\title{
Prediction and Measurement of Wall Insertion Loss
}

\author{
Thad B. Gibson and David C. Jenn, Senior Member, IEEE
}

\begin{abstract}
A propagation loss study is presented to determine the attenuation of various building walls. An approximate model was developed for the propagation of a spherical electromagnetic wave through a building wall of known material properties. This model is based on the method of images and geometrical optics. This approximate model is shown to be in good agreement with measured results.
\end{abstract}

Index Terms - Building effects, measurement, plane wave attenuation.

\section{INTRODUCTION}

$\mathbf{T}$ HIS research is part of a feasibility study for a miniature microwave powered surveillance vehicle that would be capable of operating inside buildings [1]. For this application, the reflection and attenuation (insertion loss) of a focused microwave beam by interior and exterior walls are of interest. Insertion loss measurements were taken over the frequency range of $2-6 \mathrm{GHz}$. The transmission between two identical antennas at a fixed spacing was measured to establish a reference. Then the antennas were placed on opposite sides of the wall such that the spacing was the same as for the reference measurement. A comparison of the two received powers gives an estimate of the wall insertion loss.

An approximate analytical model was developed for the transmission of an electromagnetic wave through a building wall of known material properties. This model is based on the method of images and geometrical optics. For measurements the wall is in the far field of the antenna, but not far enough to neglect the curvature of the incident wave front. The radius of curvature is large and, therefore, the wave front can be considered locally plane. This is the standard assumption for geometrical optics [2]. A locally plane approximation of a spherical wave front is used to determine the signal at the receive antenna that includes the phase and amplitude changes introduced by the wall. Based on the received signal, an equivalent free-space source can be defined and the wall removed. A similar process is used to determine a free-space image source located below the floor. Thus, these two sources radiate in free-space and their superposition yield a solution that includes both the direct and reflected transmit components. A coherent sum of the direct and reflected fields is computed in the antenna aperture. The resulting array of field points is then integrated to find the total received field of the antenna. The approximate model is shown to be in good agreement with measured results.

Manuscript received March 19, 1997; revised June 23, 1997.

The authors are with the Naval Air Warfare Center Weapons Division, Performance Analysis Branch, Point Mugu, CA 93042 USA. They are also with the Electrical and Computer Engineering Department, Naval Postgraduate School, Monterey, CA 93943 USA.

Publisher Item Identifier S 0018-926X(99)02218-8.

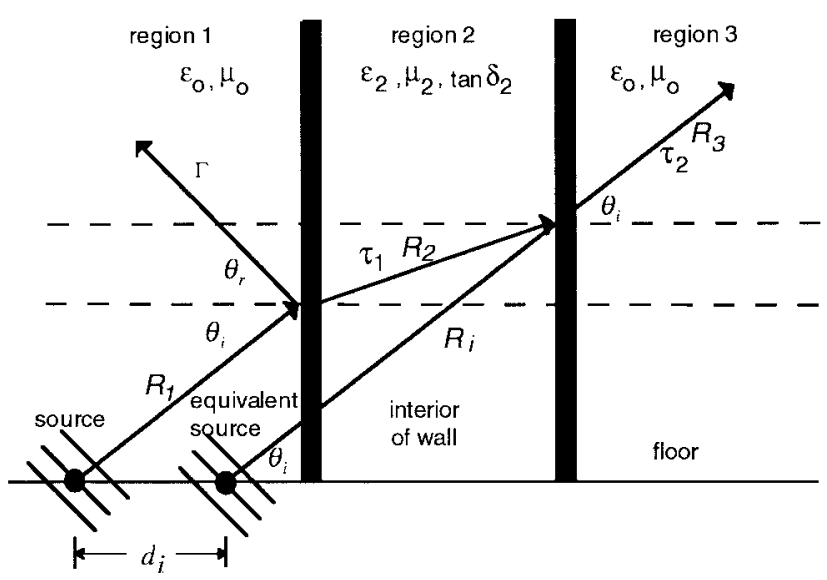

Fig. 1. Ray paths for transmission and reflection at a wall.

\section{APPRoximate Model}

The transmission and reflection of a plane wave at oblique incidence onto a panel is shown in Fig. 1. The regions on both sides of the lossy dielectric panel are assumed to be freespace. Neglecting the effects of the top and bottom edges, the behavior at each boundary is governed by the Fresnel transmission and reflection coefficients ( $\tau$ and $\Gamma$, respectively). Both the direct and floor reflected paths will be considered.

A typical direct ray path is shown in Fig. 1. Refraction causes the apparent location of the source as viewed in region 3 to move as indicated. The original source can be replaced by an equivalent source, which is located closer to the wall and modified to account for the effects of the wall. The wall can be removed and the equivalent source allowed to radiate in freespace. For the present discussion, multiple reflections inside of the wall are neglected. However, the equivalent source method can be expanded to include multiple reflections by introducing an additional equivalent source for each multiple reflection added. The electric field of the equivalent point source for the primary ray is

$$
E\left(R_{i}\right)=\frac{\tau_{1} \tau_{2}}{4 \pi R_{i}} e^{-\left(\alpha_{2} R_{2}+j\left[\beta_{0}\left(R_{1}+R_{3}\right)+\beta_{2} R_{2}\right]\right)}
$$

where $\tau_{1}$ and $\tau_{2}$ are the transmission coefficients of the two boundaries. Other quantities in (1) are the phase constant of free-space $\beta_{0}$ and the panel $\beta_{2}$ and the attenuation constant of the panel $\alpha_{2}$. The ray trajectory in region 3 can be used to determine the location of the equivalent source and, hence, the geometrical optics divergence factor $\left(1 / R_{i}\right)$.

The equivalent source radiates in the absence of the wall so that the equivalent problem is the one shown in Fig. 2. The radiation pattern of the equivalent source $E_{i}$ is given by (1). The distance $R_{i}$ is the distance from the equivalent source to a field point on the antenna grid, as indicated in Fig. 2. The 


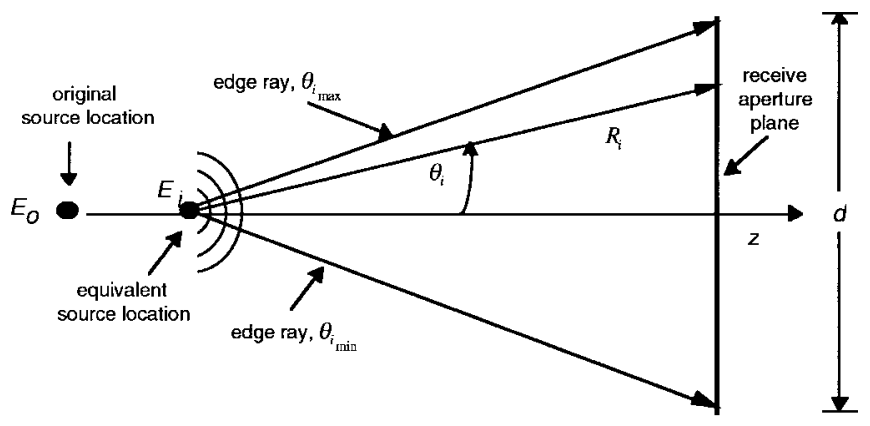

Fig. 2. Equivalent source radiating in the absence of the wall.

incident angle is stepped such that the spacing between each point on the receive aperture plane is approximately one tenth of a wavelength $(\Delta x=\Delta y \approx 0.1 \lambda)$. The range of angles $\left(\theta_{i \min }, \theta_{i \max }\right)$ are determined by the extent of the receive aperture $d$. The total received field is found by integrating over the aperture.

Using this equivalent source approach a building wall loss model was formulated. The source is located at a height $h$ above the floor and at a distance $d_{1}$ from a wall of thickness $L$. The receive location $d_{2}$ is assumed equal to $d_{1}$. The regions on either side of the wall are free-space. Given that $\theta_{i}$ is known, the distances $R_{1}, R_{2}, R_{3}$, and $R_{i}$ can be calculated. The incident angle $\theta_{i}$ is determined by discretizing the receive aperture into a grid of points, $\Delta x_{m}=m \Delta x$ and $\Delta y_{n}=n \Delta y$

$$
\theta_{i_{m n}}=\tan ^{-1}\left(\frac{\sqrt{\left(\Delta x_{m}\right)^{2}+\left(\Delta y_{n}\right)^{2}}}{d_{1}+L+d_{2}-d_{i}}\right) \text {. }
$$

In determining the received field for a vertically polarized antenna, only the vertical component is required. Therefore, the electric field of the equivalent source includes a polarization factor

$$
\begin{aligned}
E_{d}\left(R_{i}\right)= & \frac{\left(1-\sin ^{2} \theta \cos ^{2} \phi\right) \tau_{1} \tau_{2}}{4 \pi R_{i}} \\
& \times e^{-\left(\alpha_{2} R_{2}+j\left[\beta_{0}\left(R_{1}+R_{3}\right)+\beta_{2} R_{2}\right]\right)} .
\end{aligned}
$$

Equation (3) is the equivalent spherical source that radiates in the absence of the wall.

The floor reflection is included using an equivalent image source. The location of the image is directly below the source. Since the floor is not a perfect conductor, the image is not of equal intensity, but approximately reduced by a factor $\Gamma_{r}$-the reflection coefficient of the floor. With the image present, the floor is removed while the wall remains. In general, this step is not entirely accurate, because it assumes that rays hitting the wall below floor level pass through the wall medium. However, for the geometry under consideration (equal antenna heights and equal distances from the wall) the approximation is accurate.

Similar to the direct path, the original source is traced to the receiving antenna to determine the maximum and minimum angles of incidence of the reflected rays, $\theta_{r \min }$ and $\theta_{r \max }$. The rays corresponding to the maximum and minimum angles of incidence are traced back to the point of intersection at $d_{r_{x}}$ and $d_{r_{z}}$, as shown in Fig. 3. This is the location of the

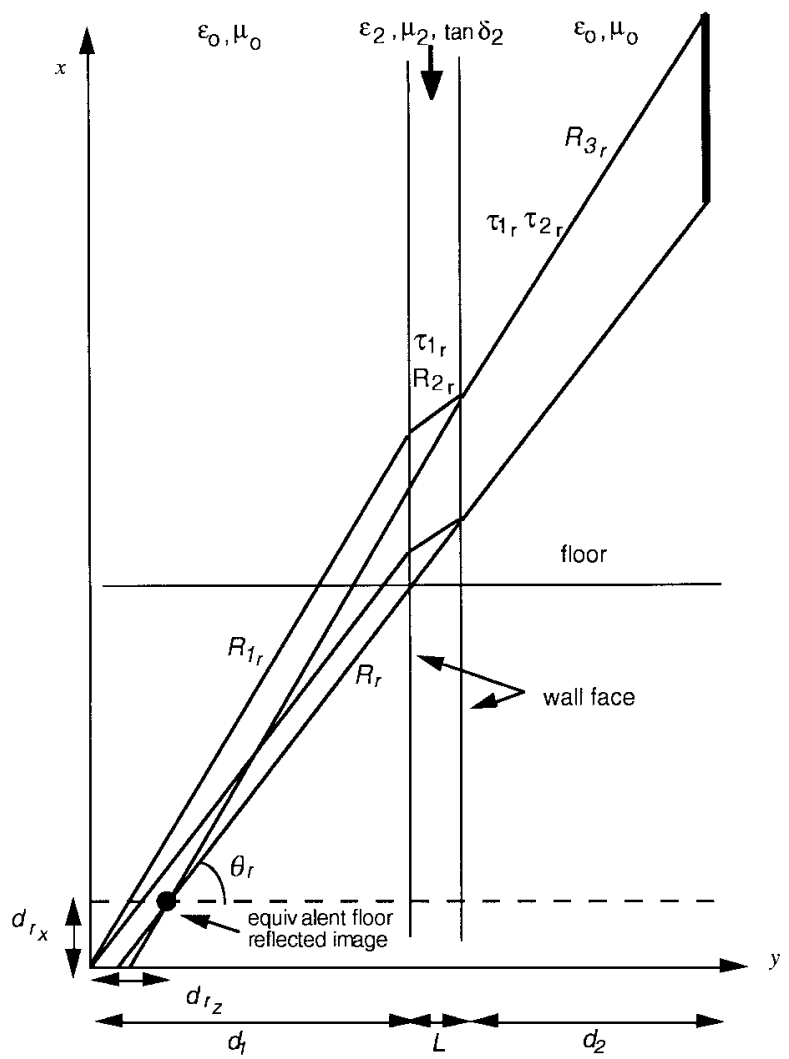

Fig. 3. Ray path for the reflection based on a source image located below the floor.

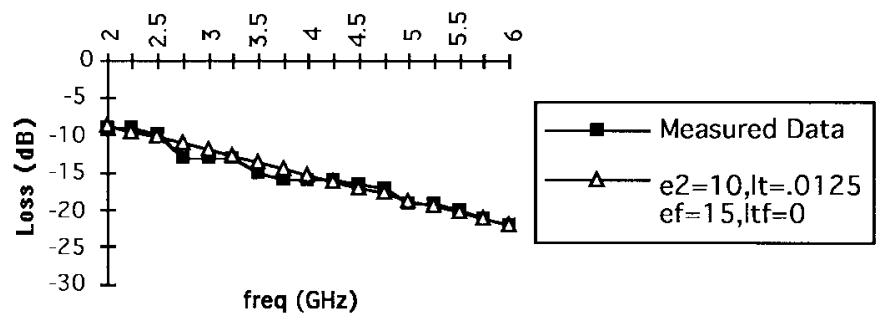

Fig. 4. Comparison of measured and computed insertion loss for a 1-ft-thick concrete wall.

equivalent floor reflected source. Due to asymmetry, the lines will intersect off of the $z$ axis. The geometrical relationships are similar to those for the direct rays. Given that $\theta_{r}$ is known the distances $R_{1 r}, R_{2 r}$, and $R_{3 r}$ can be calculated. The total field resulting from the reflected rays is given by

$$
\begin{aligned}
E_{r}\left(R_{r}\right)= & \frac{\left(1-\sin ^{2} \theta \cos ^{2} \phi\right) \Gamma_{r} \tau_{1_{r}} \tau_{2_{r}}}{4 \pi\left(R_{r}\right)} \\
& \times e^{-\left(\alpha_{2} R_{2_{r}}+j\left(\beta_{0}\left(R_{1_{r}}+R_{3_{r}}\right)+\beta_{2} R_{2_{r}}\right)\right) .}
\end{aligned}
$$

The coherent sum of the direct and reflected rays are used to determine the total field at each aperture point. These are integrated to obtain the total received power $E_{T}$.

\section{COMPuTED AND MeAsured Data}

The insertion losses of various walls were measured by comparing the free-space transmission between two antennas to those with walls located at the midpoint between the 


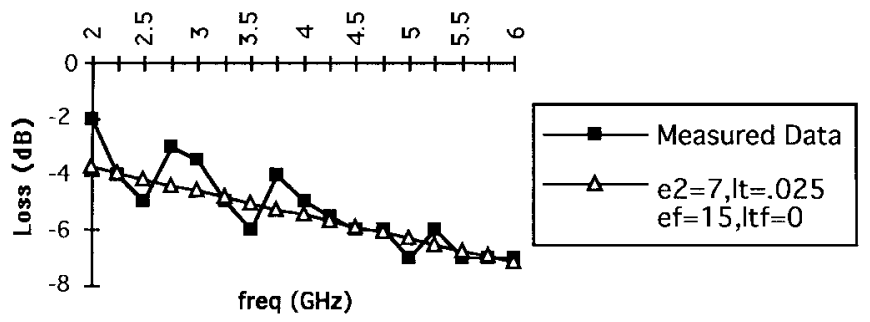

Fig. 5. Comparison of measured and computed insertion loss for double-wide wood doors.

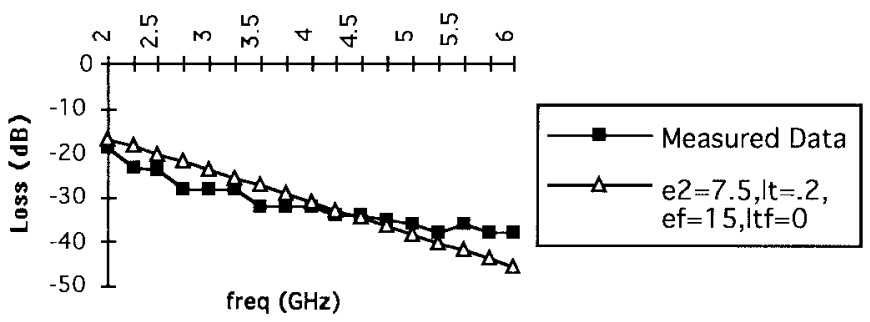

Fig. 6. Comparison of measured and computed insertion loss for metal doors.

antennas. The measurement was also simulated using the analytical model. The received power was calculated for a unobstructed path, then compared to the received power when a panel was inserted. Initial values for the constitutive parameters of the wall were obtained from handbooks. In general, a small modification of the initial value was sufficient to give good agreement with the measured results. A typical set of data is shown in Figs. 4-6. A complete set is available in [3]. The measured data was taken from various locations in Spanagel Hall, Naval Postgraduate School, Monterey, CA. The computed data is shown for the dielectric constant and loss tangent of the wall $\left(e_{2}, l_{t}\right)$ and the floor $\left(e_{f}, l_{t f}\right)$, respectively.

Fig. 4 shows the loss for a thick cement wall. Because of the high density of the cement, the loss is mainly due to reflection at the wall/air interface. Fig. 5 shows the insertion loss for double-width wood doors. The ripple in the measured data is due to reflections from the vertical part of the door frame (which is metal). The peaks in the ripple correspond exactly to those predicted using a simple multipath model. Finally, Fig. 6 gives the insertion loss of 1.75 in metal doors.

\section{Summary AND CONCLUSIONS}

A mathematical model was developed to approximate the propagation of an electromagnetic wave through a wall. The assumptions inherent in the model are the following.

1) The polarization of the source and receive antennas is vertical.
2) Surface roughness of the wall is neglected and the faces are parallel.

3) Loss tangent and dielectric are constant throughout the wall.

4) A frequency independent dielectric constant and loss tangent are used in the equations for impedance, phase, and attenuation.

5) Multiple reflections within the wall are neglected.

Discrepancies between the measured and computed data can be attributed to the limitations of the model and possible inaccuracies in the measured data. This simple model provides a relatively accurate estimate of wall losses if the dielectric constant and loss tangent can be determined from visual inspection of the wall.

\section{REFERENCES}

[1] D. Jenn, R. Vitale, G. Lee, and T. Gibson, "Microwave powered microRPV," in Proc. 1st Int. Conf. Emerging Technol. Micro Air Veh., Georgia Inst. Technol., Atlanta, GA, Feb. 19-20, 1997.

[2] D. C. Jenn, Radar and Laser Cross Section. New York: Amer. Inst. Aeronaut. Astronaut. (AIAA), 1995.

[3] T. B. Gibson, "Propagation loss study and antenna design for the micro-remotely powered vehicle (MRPV)," Master's thesis, Naval Postgraduate School, Monterey, CA, Sept. 1995.

Thad B. Gibson received the B.S. degree in electrical engineering from the University of Texas at El Paso and the M.S. degree in electrical engineering from the Naval Postgraduate School, Monterey, CA, in 1990 and 1995, respectively.

Since 1990 he has worked as a Systems Analyst for various air-to-air missile systems at the Naval Air Warfare Center Weapons Division (NAWCWD), Point Mugu, CA. His current work includes the analysis and performance assessment of complex weapon systems using flight test, Hardware-In-theLoop (HIL), and six degree of freedom (6-DOF) computer simulations.

Mr. Gibson is a member of Eta Kappa Nu. In 1994, he received a NAWCWD Fellowship for graduate studies in electronic systems engineering specializing in electromagnetic modeling and antenna design.

David C. Jenn (S'75-M'82-SM'93) received the Ph.D. degree in electrical engineering from the University of Southern California, Los Angeles, in 1987. From 1976 to 1978, he was with McDonnell Douglas Astronautics Co., where he was involved in the design of small arrays and radomes for airborne platforms. In 1978 he joined Hughes Aircraft Co., where he concentrated on the design and analysis of high-performance phased-array antennas for radar and communication systems. In 1990 he joined the Department of Electrical and Computer Engineering at the Naval Postgraduate School as an Associate Professor. Since then he has continued his work on the application of the method of moments to array and reflector antenna design, as well as scattering by complex bodies. He is author of Radar and Laser Cross Section Engineering (American Institute of Aeronautics and Astronautics (AIAA): New York, 1995).

Dr. Jenn is a Senior member of the American Institute of Aeronautics and Astronautics (AIAA), Tau Beta Pi, American Society for Engineering Education (ASEE), and the Applied Computational Electromagnetics Society (ACES). 